\title{
Undergraduate education and training in optics at the Universidad Nacional de La Plata
}

\section{Mario Garavaglia}

Mario Garavaglia, "Undergraduate education and training in optics at the Universidad Nacional de La Plata," Proc. SPIE 3831, Sixth International Conference on Education and Training in Optics and Photonics, (16 June 2000); doi: 10.1117/12.388737

Event: Education and Training in Optics and Photonics (ETOP'99), 1999, Cancun, Mexico 


\title{
Undergraduate education and training in optics at the Universidad Nacional de La Plata
}

\author{
Mario Garavaglia \\ Departamento de Física, Universidad Nacional de La Plata \\ and \\ Centro de Investigaciones Opticas \\ CC 124 Correo Central, 1900 La Plata, Argentina \\ E-mails: garavagliam@ciop.unlp.edu.ar \\ laserlab@pinos.com
}

\begin{abstract}
After few historical and statistical data related to the Universidad Nacional de La Plata, follows a description of activities on undergraduate training in Optics at the Laboratorio de Optica, belonging to the Faculty of Astronomical and Geophysical Sciences, and on undergraduate education in Optics at the Departamento de Física, belonging to the Faculty of Exact Sciences.
\end{abstract}

Keywords: Undergraduate education, undergraduate training, optics at universities

\section{INTRODUCTION}

Argentine is a southamerican federal country divided in 23 provinces and a Federal Capital, the Buenos Aires city. Until November 19, 1882, the Federal Government of Argentina and the government of the Province of Buenos Aires were established in the Buenos Aires City. In 1881 it was decided to found La Plata City as the capital of the Province of Buenos Aires. At that time the research activity in the Province of Buenos Aires was mostly related to Natural Sciences and Astronomy. In fact, in 1877 was established the Museum of Natural Sciences and, at the beginning of 1882 , was initiated the research activity in Astronomy to observe the transit of Venus in front of the solar disk on December 4, 1882. The first director of the Museum was the argentine naturalist $\mathrm{P}$. Moreno, while the director of the astronomical activities was the French astronomer C. Beuf. These scientific activities together with other academic and professional activities were concentrated from 1905 in the Universidad Nacional de La Plata. In the same year was also established the Departamento de Física, whose first director was the German physicist E. Bose.

The actual academic structure of the Universidad Nacional de La Plata is formed by fifteen faculties: Humanities, Fine Arts, Natural Sciences, Exact Sciences, Astronomy and Geophysics, Engineering, Architecture, Medicine, Veterinary, Agriculture, Law, Economy, Odontology, Periodism and Informatics. Also belong to the University the Museum of Natural Sciences, the University Radio (1923), and the Computer Centre (1960). Besides, three Secondary Schools, one of them oriented to Fine Arts and a Pilot Primary School complete the academic structure of the Universidad Nacional de La Plata. 80.000 students are at the Universidad Nacional de La Plata.

Scientific and technical activities on Optics are mainly concentrated in the Faculty of Exact Sciences, and of Astronomy and Geophysics. Vision and Ophthalmic optical studies are mostly located at the Faculty of Medicine, while Colour studies related to fine arts and design teaching are located at the Faculty of Fine Arts.

\section{THE OPTICAL LABORATORY AT THE FACULTY OF ASTRONOMY AND GEOPHYSICS}

The Optical Laboratory at the Faculty of Astronomy and Geophysics yearly offers an optional course on Astronomical Optics to students of Astronomy in the fourth academic year of the five-year "Licenciado en Astronomía" career. The main subjects are related with the image quality yielded by a complex optical instrument. Then, students learn on optical glass 


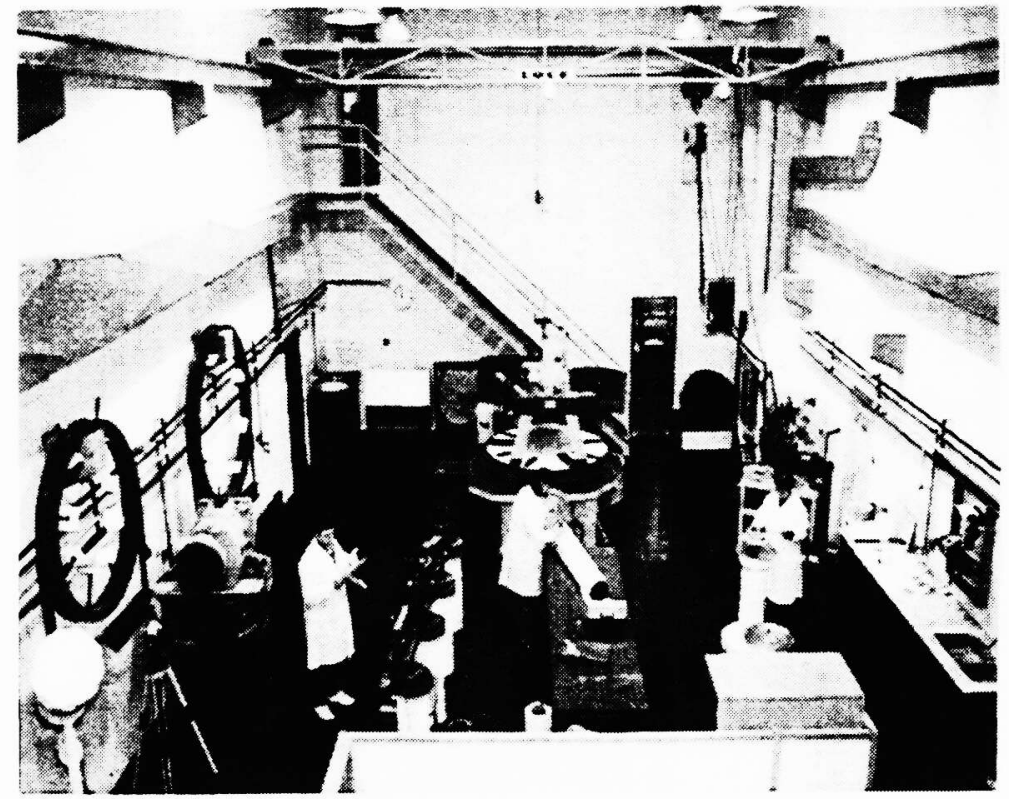

Figure 1. General view of the optical workshop

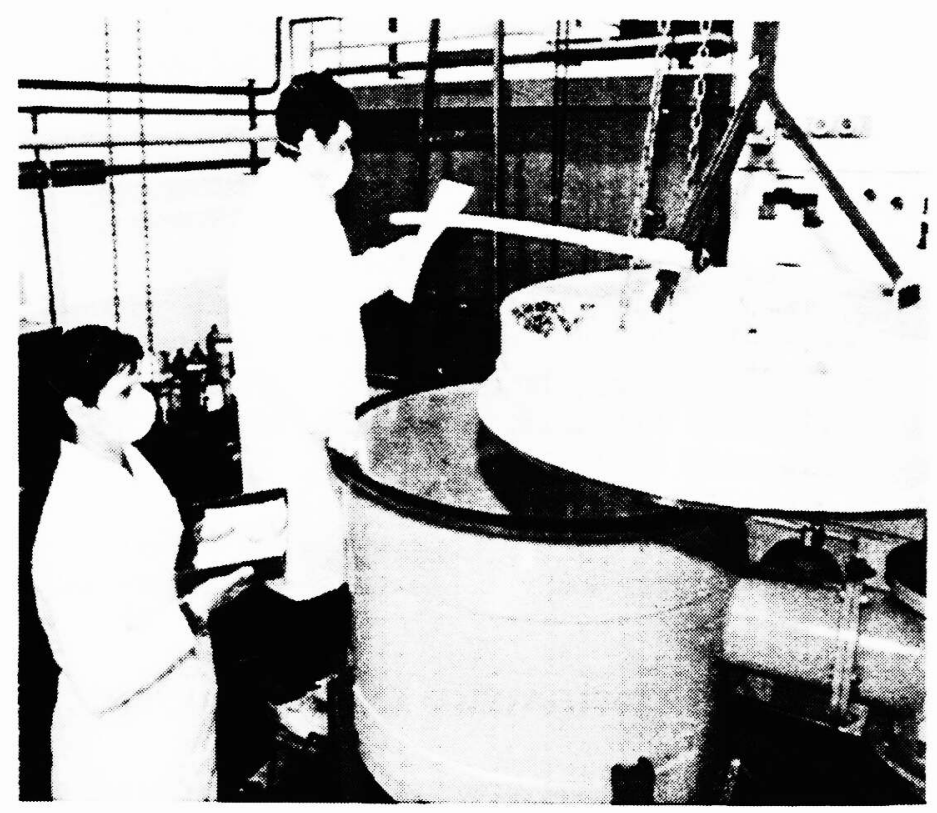

Figure 3. Vacuum deposition facility

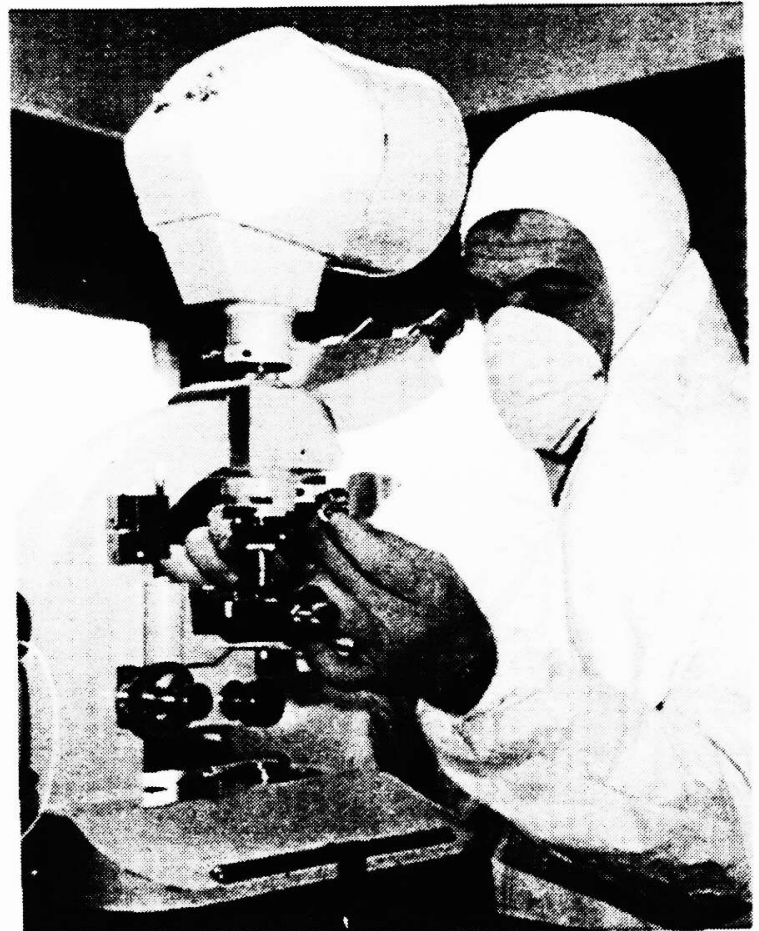

Figure 2. Repairing a microscope

properties: third. fifth and higher orders aberrations theory; refraction and reflection telescopes: spectrographs: interferometers: CCD cameras: atmospheric seeing, and active and adaptive optics. $70 \%$ of the course is dedicated to theoretical explanations and problems resolution. The experimental activity is divided between the optical workshop practices and observations with $43 \mathrm{~cm}$ and $80 \mathrm{~cm}$ aperture telescopes.

The Optical Laboratory is also responsible to conduct an optional course on Optical Instruments devoted to students that prefer the technical aspects related with optical materials, optical design, tools and polishing techniques, vacuum deposition techniques, optical testing and laboratory quality management. $70 \%$ of the course is dedicated to practical activities at the optical workshop, optical testing laboratory, and to repair optical instruments the Optical Laboratory receives from different laboratories inside the university and also from outside. This last activity is very important because students are oriented to make technical optical services to the community. To attend this optional course on Optical Instruments the Optical Laboratory also admits technicians and vocational students. Along the course they can build-up a home reflector telescope. Figures 1,2 and 3 are general view of the optical workshop. the laboratory for repair optical instruments and the vacuum deposition facility. 


\section{DEPARTMENT OF PHYSICS OF THE FACULTY OF EXACT SCIENCES}

The Department of Physics of the Faculty of Exact Sciences is responsible to teach Physics at two levels: Ten academic semester's undergraduate courses to reach the "Licenciado" degree in Physics, and postgraduate courses plus the Doctoral Thesis to reach the Doctor degree in Physics. After five semesters of courses on Algebra, Calculus. Geometry. General Physics. Analytical Mechanics and Electromagnetic Theory students must choose nine optional subjects from a menu that contains some fifty offered courses. Students attend optional subjects from the sixth to eighth semesters. Figure 4 is a picture of the Department of Physics building at 1926: at that time its director was the German physicist R. Gans.

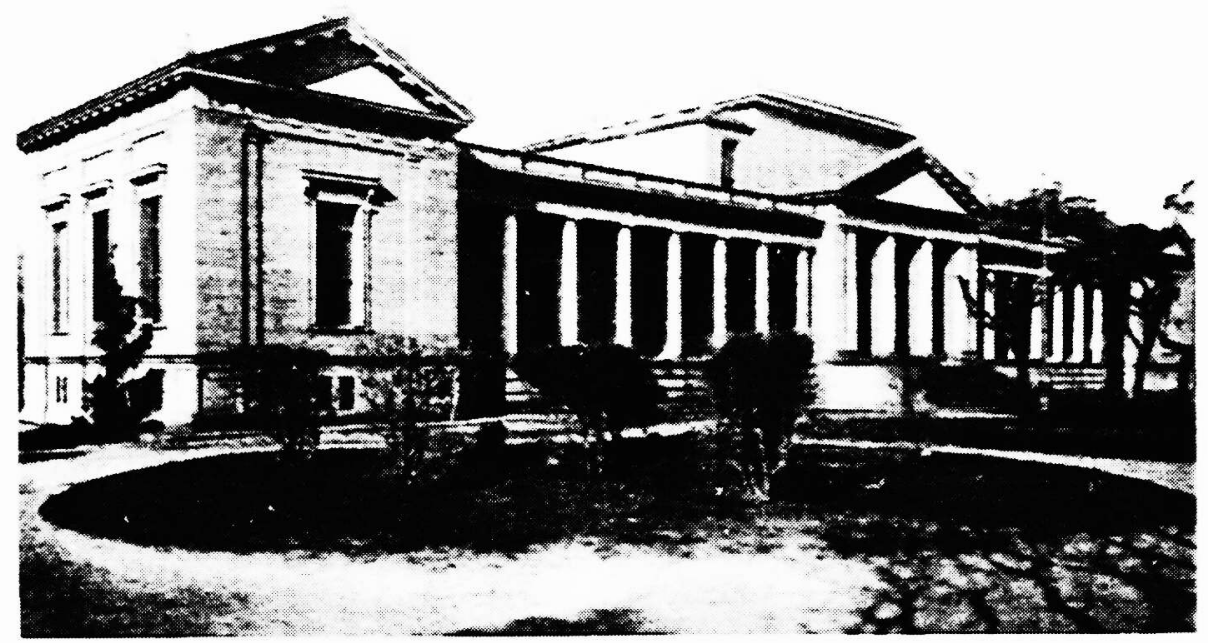

Figure 4. La Plata Department of Physics building at 1926. It resembles the building of the Jena Institute of Physics, Germany.

In this Section a briefly description of recent results obtained by students in three of the optional subjects will be done. Referred optical subjects are Instrumental Optics, Introduction to Fourier Optics. and Introduction to Coherence. The age of students ranges from 20 to 22 years old. A one-semester period is employed to develop the theoretical approach to the problem, to conceive a model for laboratory purposes, to make experiments, and to discuss results obtained. At the end of the semester students present a formal report on their activity Very often such a report contains very interesting results and is taken as base for a scientific manuscript. which is sending to the annual Argentinean meeting on Physics. Regularly all papers authored by students are presented by themselves. If results obtained by students are clearly original, they used some more extra time to write a scientific paper, which is sending for publication or to be presented in an international meeting. In a few cases. students themselves attended those international meetings and discussed their results with participants. Comments on three theoretical/experimental typical works using different arrays of the Young's interferometer are presented.

\subsection{Instrumental Optics: Study of Retardation Plates ${ }^{1}$}

In many scientific and technologic measurements the coherence of the light is essential to perform good observations and to get the best results. The same can be said of its polarisation state. because different type of transparent materials can affect its propagation through the optical device. Association of both light features -coherence and polarisation states- appears in some interference experiments, in which polarizers and retardation plates are employed. A question arises when retardation plates are employed to change the optical path length in the experiment -producing a proportional displacement of interference fringes- and rotated around an axis perpendicular to them to change the polarisation state of the light -producing a proportional change in the visibility of fringes-. The answer is that the interference fringes change their visibility according to the change of the polarisation state of the light, and also they rotate according with the rotation angle of the retardation plate

To measure this last effect a Young's apparatus whose $1 \mathrm{~mm}$ wide slits are $36 \mathrm{~mm}$ apart was used. Interference fringes were observed by using a 40x-magnification microscope placed at $6.7 \mathrm{~m}$ from the plane of slits. The experimental set up is depicted in Figure 5. Interferograms were recorded on Kodak E $100 \mathrm{SW}$ colour film. Regular Young fringes parallel to the apparatus slits are observed, but according with the rotation of the retardation plate mounted in front of one of the slits, they became inclined to right or to left. diminishing the distance $d$ between fringes. Also, the visibility of fringes is strongly modified. This effect was measured and analysed. and it is attributed to the fact that rotations of a retardation plate make the beam propagated through it to follow a conical propagation after emerging it ${ }^{2}$. The position of the beam along the circumference corresponding to some normal sectioning of the cone is proportional to the rotation angle of the retardation 


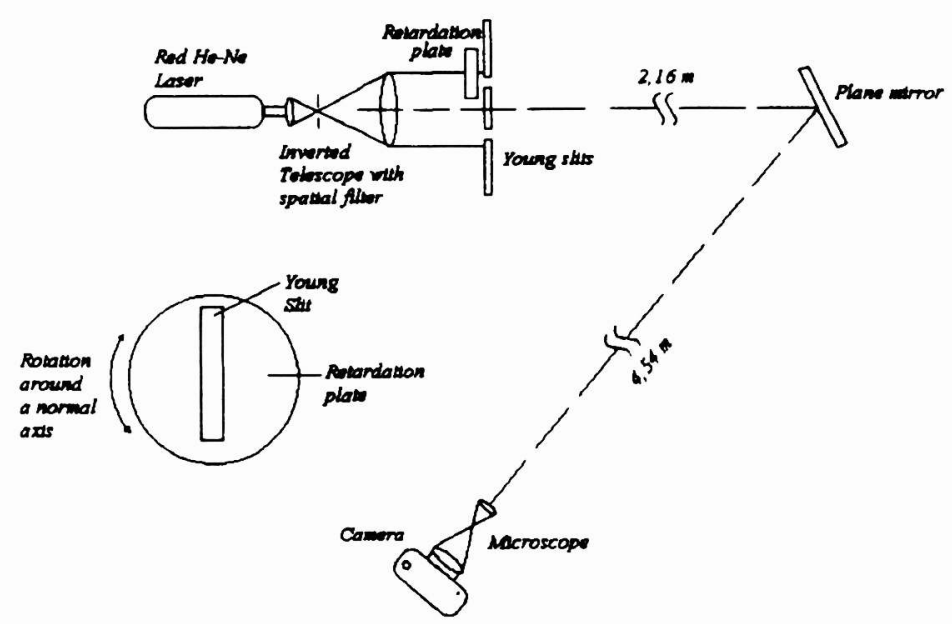

Figure 5. Young's interferometer set-up

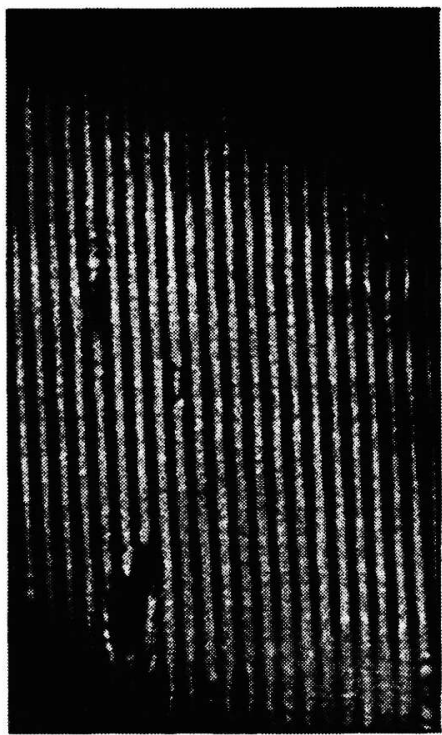

$d=1.506 \mathrm{~mm}$

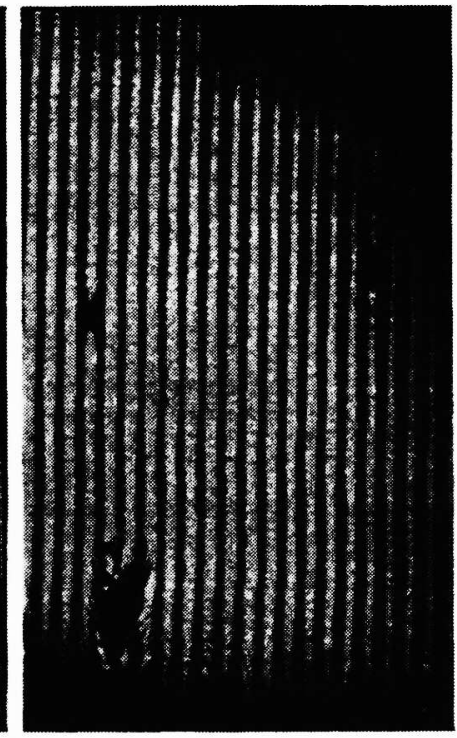

$1.535 \mathrm{~mm}$ plate. Figure 6 shows interference fringes corresponding to a $\lambda / 4$ retardation plate placed in front to one of the Young's slit. Interferograms were recorded at four angular positions of the retardation plate; each of them corresponds to a $\pi / 2$ rotation from the previous position. Below to each interferogram is stated the respective average distance $d$ between fringes. Figure 7 shows interferograms as in Figure 6 corresponding to a $\lambda / 2$ retardation plate. In this case, the radius of the circumference of the section of the propagation cone is large enough that laser light from both slits did not overlap each other in certain range of the angular rotation of the plate. Then, no interference fringes are recorded at all under these conditions This effect appears in the entire observed field in the last picture from the left at Figure 7 . It is also partially observed in the other three pictures at Figure 7 , but at different places according with the rotation of the plate.

Figure 6 . Interference fringes corresponding to a $\lambda / 4$ retardation plate

A practical conclusion brings this experimental study. Before making an experiment that includes retardation plates, it is necessary to test them under rotation around their geometric axis to avoid difficulties in interpreting experimental results and their uncertainties.

\subsection{Introduction to Fourier Optics: Young Interference Experiments with a Laser Filamentary Source ${ }^{3}$}

Young's experiments very often are performed using two-slit plate instead a two-pinhole plate. However, M. Born and E. Wolf ${ }^{4}$ made an important remark with respect to the replacement of pinholes by narrow parallel slits in Young's experiments. They stated that "in this way the intensity of the patterns can be increased, though there is an additional experimental difficulty in securing correct orientation of the source". That is, the slit source must be parallel to the narrow pair of Young's slits. However, the consequences of not keeping the slits and the source parallel were explored, as a formal extension of the traditional Young set up. The interference pattern produced by a long parallel slits illuminated by a 


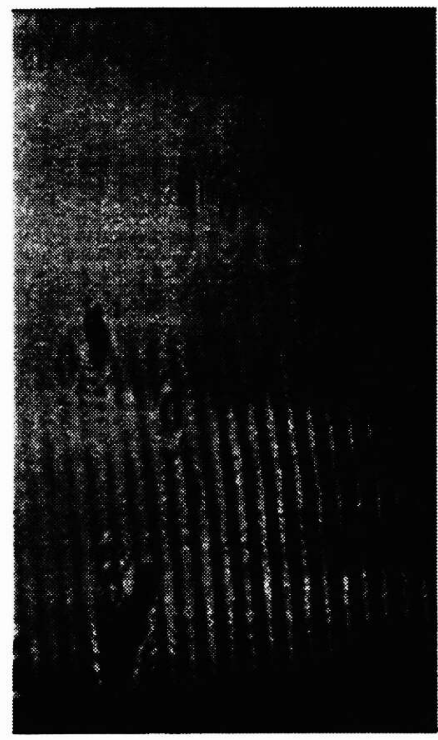

$d=1.487 \mathrm{~mm}$

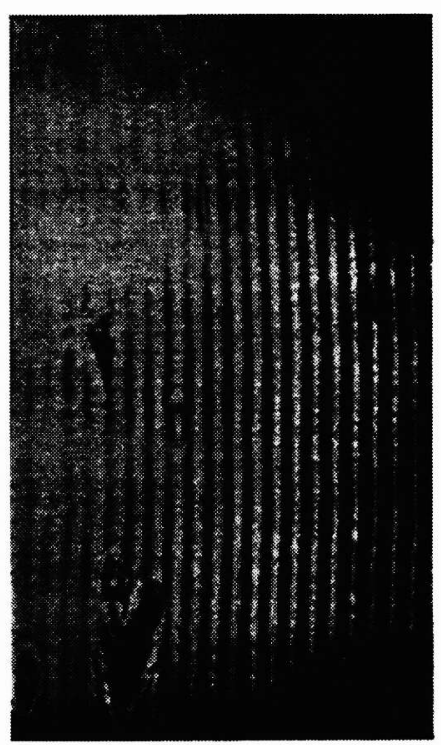

$1.538 \mathrm{~mm}$

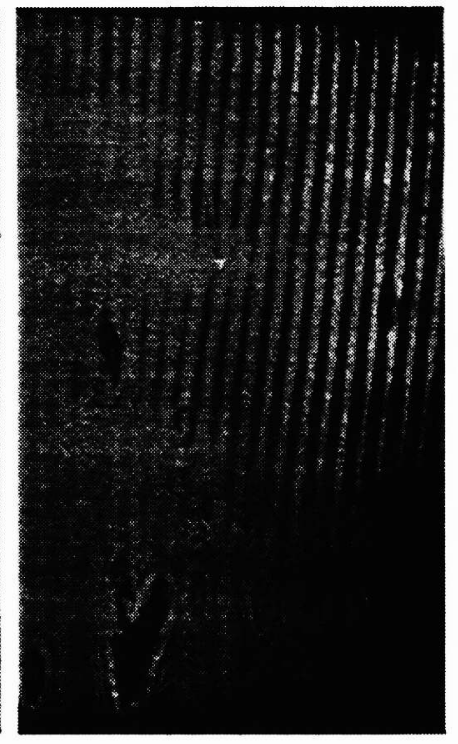

$1.507 \mathrm{~mm}$

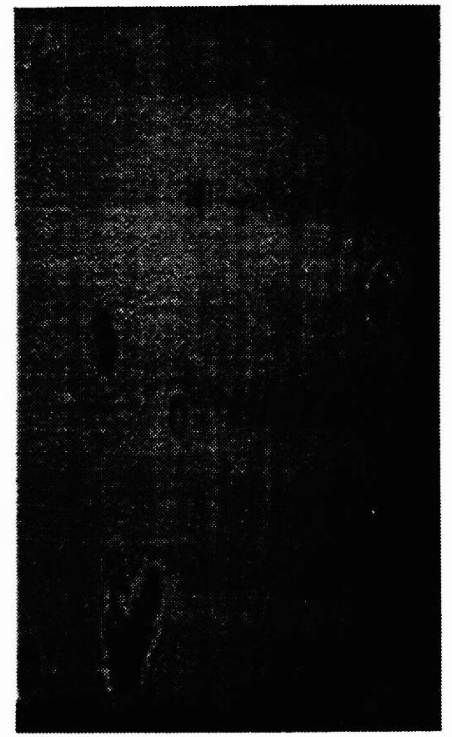

$-\cdot-$

Figure 7 . Interference fringes corresponding to a $\lambda / 2$ retardation plate

filamentary monochromatic light source was observed. and the effect of the rotation of the slits while the source remains in the same position will be described. When the filamentary light source is rotated in a plane parallel to that of the slits. the visibility of the interference fringes is always extremely high. but their geometrical distribution and the distance among them depend upon the rotation angle and the observation distance.

In the experimental set up sketched in Figure 8, the beam of a red He-Ne laser $(\lambda=633 \mathrm{~nm})$ was expanded and collimated to about $5 \mathrm{~cm}$ in diameter. The plane face of a cylindrical lens was aligned perpendicularly to the beam. The Young's slits were $37.5 \mu \mathrm{m}$ wide and $237.5 \mu \mathrm{m}$ apart: their length was $22 \mathrm{~mm}$. Slits were aligned parallel to the focal line, $25 \mathrm{~cm}$ away from it. on a graduated rotator mounting. Photographs were taken with Kodak E $100 \mathrm{SW}$ colour film on a lensless camera at different distances $R$ to the slits. at each of which the slits were rotated from $0^{\circ}$ to $90^{\circ}$.

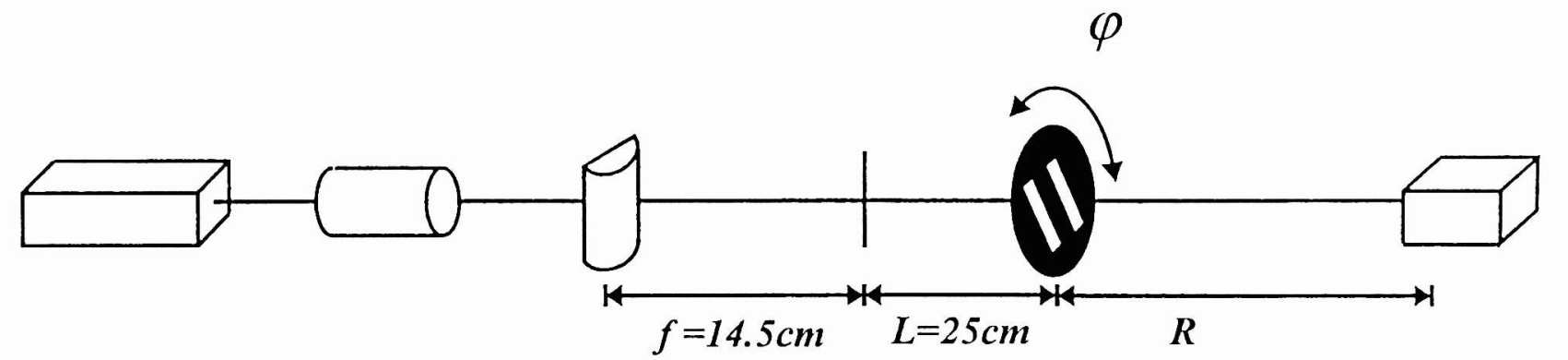

He-Ne laser

Collimator Cylindrical lens

Focal line

Young slits

Lensless camera

Figure 8. Young's interference experimental set up with a laser filamentary source

The proposed model is sketched in Figure 9 . The laser filamentary source is placed according with coordinates $\left(x_{f} ; y_{f}\right)$, while the two-slit plate of the Young's interferometer is placed with $\left(x_{s} ; y_{s}\right)$ and rotated through $\varphi$. The observation plane at coordinates $\left(x_{0} ; y_{0}\right)$ is placed at different distances $R$ from the two-slit plate. Is in these planes of coordinates $\left(x_{0} ; y_{\alpha}\right)$ where 


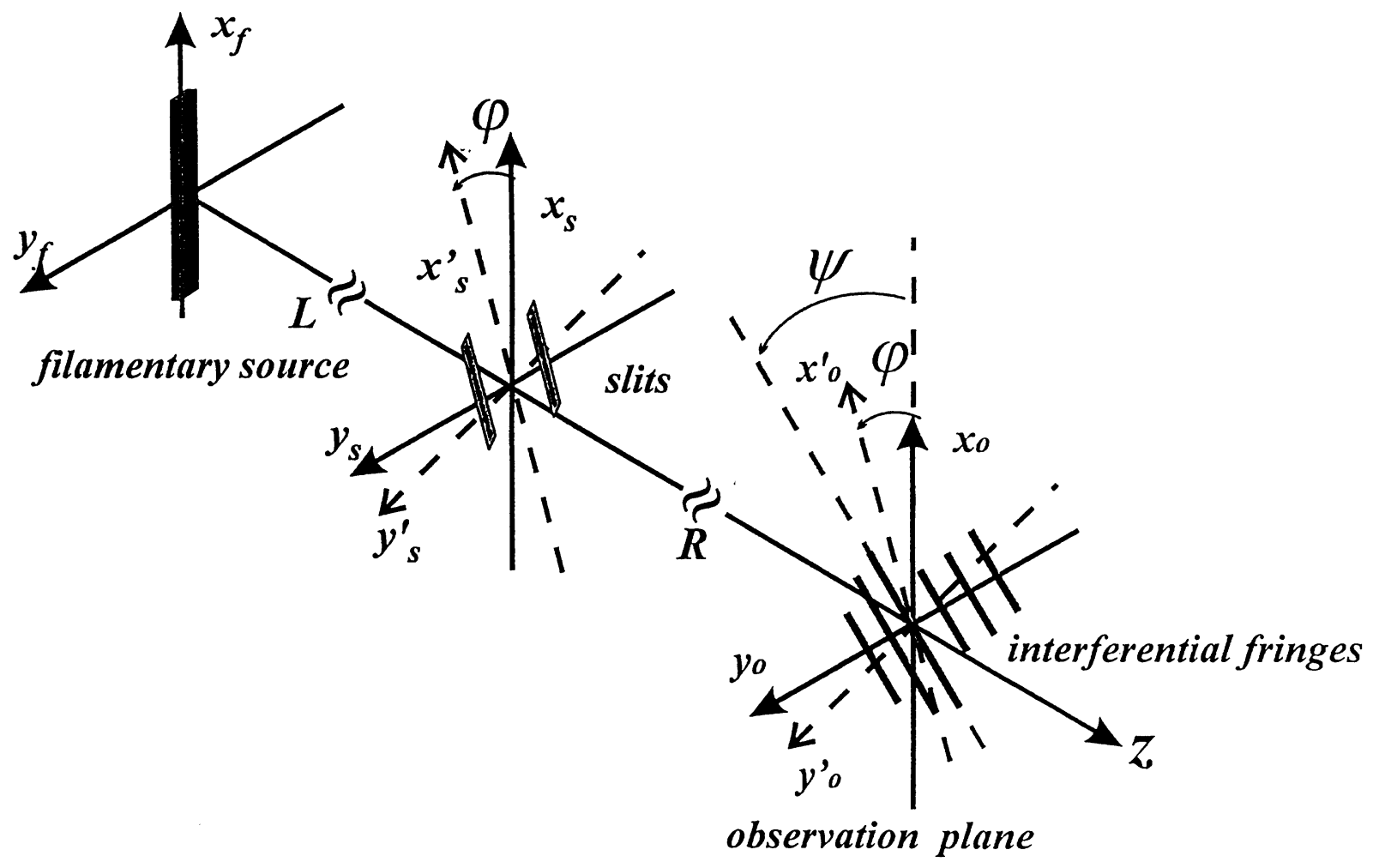

Figure 9. Proposed model to investigate Young's interference pattern produced by a laser filamentary source

the photographic plate is placed to record Young interference fringe patterns as a function of $\varphi$ and $R$. The field amplitude distribution in the rotated coordinates $\left(x_{o}^{\prime} ; y_{a}^{\prime}\right)$ is equal to:

$$
A\left(x_{o}^{\prime}, y_{o}^{\prime}\right)=\frac{1}{\sqrt{L / R+\sin ^{2} \varphi}} \cos \frac{\pi a}{\lambda R}\left[\frac{\sin \varphi \cos \varphi}{\sqrt{L / R+\sin ^{2} \varphi}} x_{o}^{\prime}-y_{o}^{\prime}\right] \text {, }
$$

whose sinusoidal variation means that interference fringes of the form:

$$
y_{o}^{\prime}=\frac{\sin \varphi \cos \varphi}{L / R+\sin ^{2} \varphi} x_{o}^{\prime}+n \frac{2 \lambda R}{a}
$$

or, in terms of $x_{o}$ and $y_{0}$, of the form:

$$
y_{o}=(1+R / L) \tan \varphi x_{o}+n \frac{2 \lambda R}{a} \frac{\left(1+(R / L) \sin ^{2} \varphi\right)}{\cos \varphi}
$$

can be observed where $n=0, \pm 1, \pm 2, \ldots$

The slope of this equation is:

$$
\tan \psi=(1+R / L) \tan \varphi,
$$

where $\psi$ is the angle between the interference fringes and the filamentary source. 


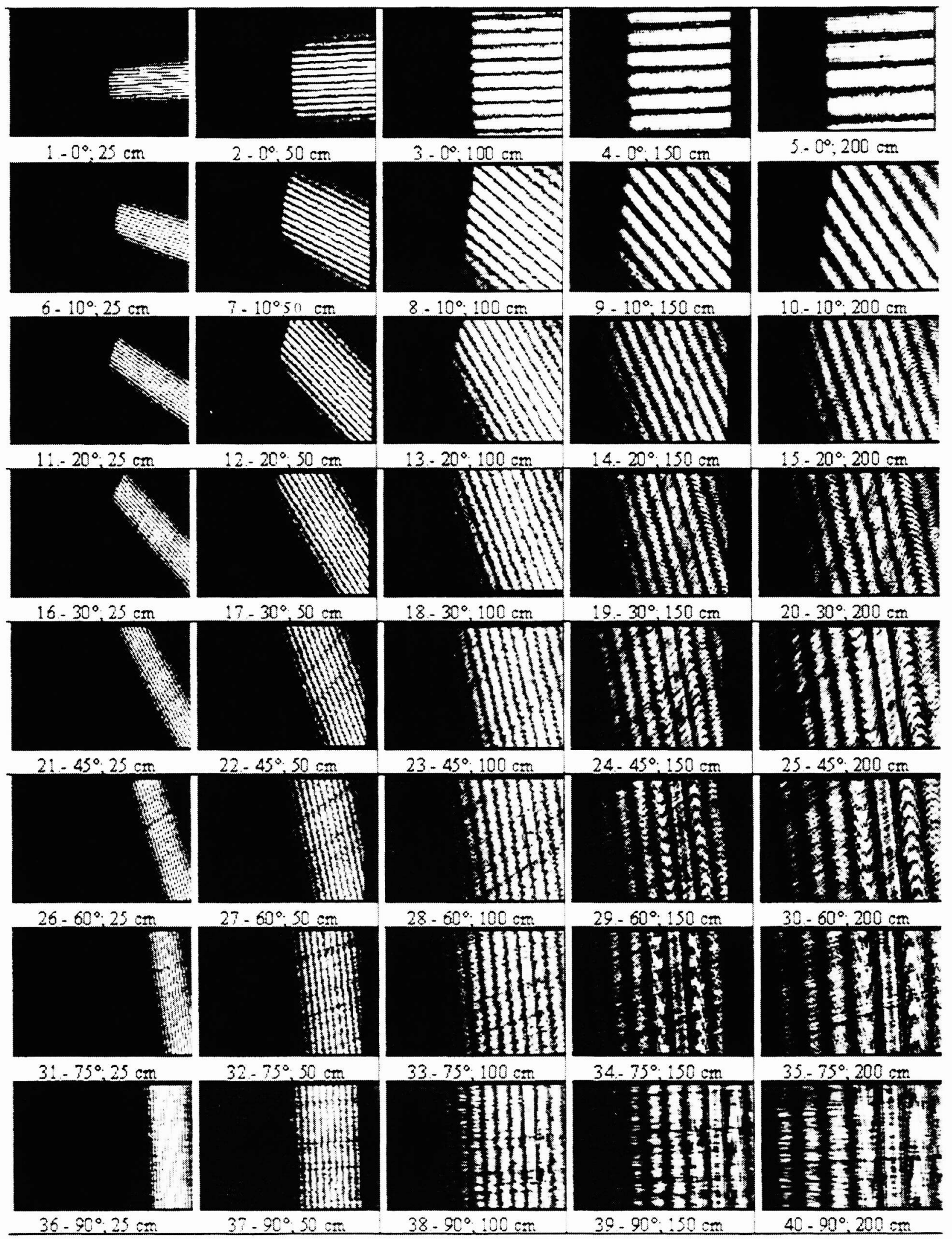

Figure 10. Interferograms for a monochromatic coherent filamentary source, taken at different observation distances with different rotations of the slits 
Besides, the position of the first interference fringe $n=1$ in each reference system for $x_{o}=0$ and $x_{o}^{\prime}=0$, is:

$$
y_{o}=\frac{2 \lambda R}{a} \frac{\left(1+(R / L) \sin ^{2} \varphi\right)}{\cos \varphi},
$$

and:

$$
y_{o}^{\prime}=\frac{2 \lambda R}{a}
$$

respectively, while the distance $d$ between two consecutive fringes is:

$$
d=\frac{2 \lambda R}{a} \cos (\psi-\varphi)
$$

Experimental results coincide remarkably well with the stated theoretical model. In fact, Figure 10 contains a collection of 40 examples of interferograms. They were photographed at five different values of the ratio $R / L$ and at eight angles $\varphi$. The angle $\psi$ was measured in them, and is plotted against $\varphi$ in Figure 11. The angular factor $\cos (\psi-\varphi)$ is plotted against the angle $\varphi$ in Figure 12, taking into account the experimental values adopted for the distance $R$ and the rate of distances $R / L$. The ordinate axis in Figure 12 is normalised, in the sense that $d=2 \lambda R / a$ for $\varphi=0$.

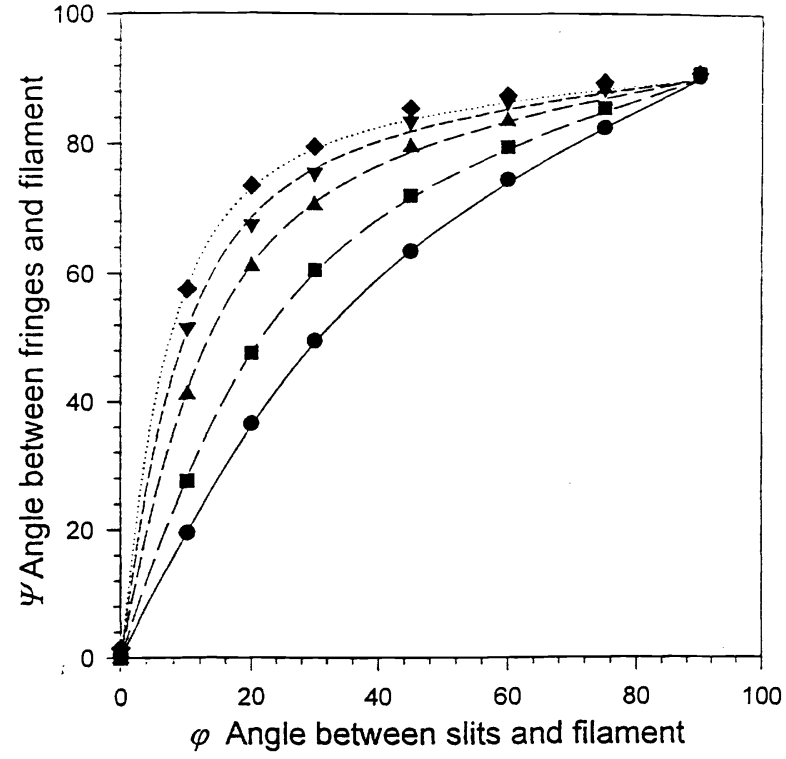

Figure 11. $\psi$ plotted against $\varphi$

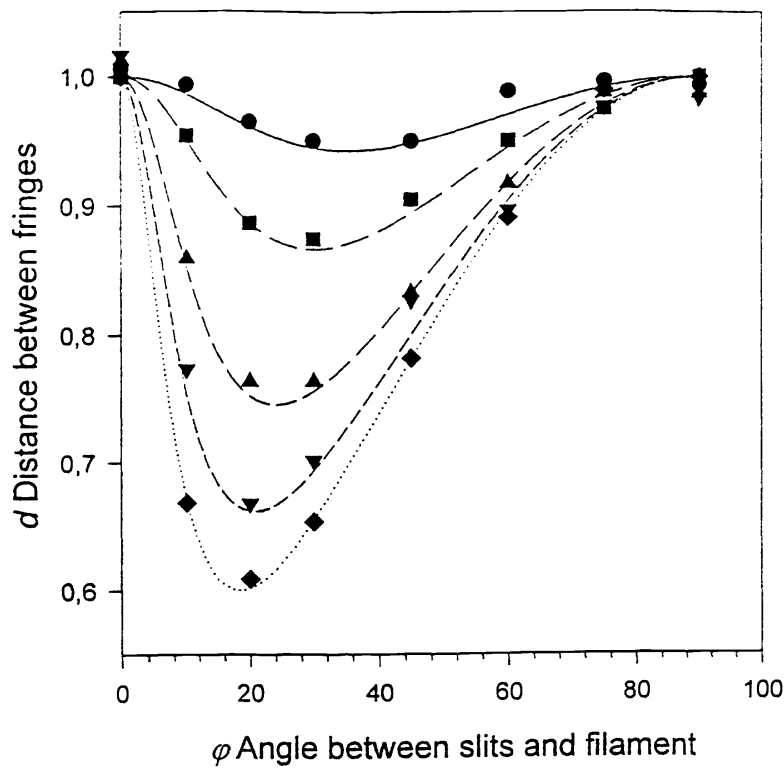

Figure 12. $d$ plotted against $\varphi$

\subsection{Introduction to Coherence: Young Interference Experiments with an Incoherent and Quasimonochromatic Filamentary Source ${ }^{5}$}

As a natural extension of the observed interferograms with a laser filamentary source ${ }^{3}$ described in Section 3.2., it was performed theoretical and experimental studies in case of an incoherent and quasimonochromatic filamentary source. The experimental set-up is similar to that at Figure 8, but the filamentary source is the linear filament of an incandescent lamp. It is almost seven times larger than the pair of slits. A red filter centred at $\lambda=635 \mathrm{~nm}$ and $\Delta \lambda=150 \mathrm{~nm}$ full width was placed in front of slits. The observation of interference patterns was performed in a fixed plane using a photographic camera. Figure 13 is a picture of the actual experimental set-up. 


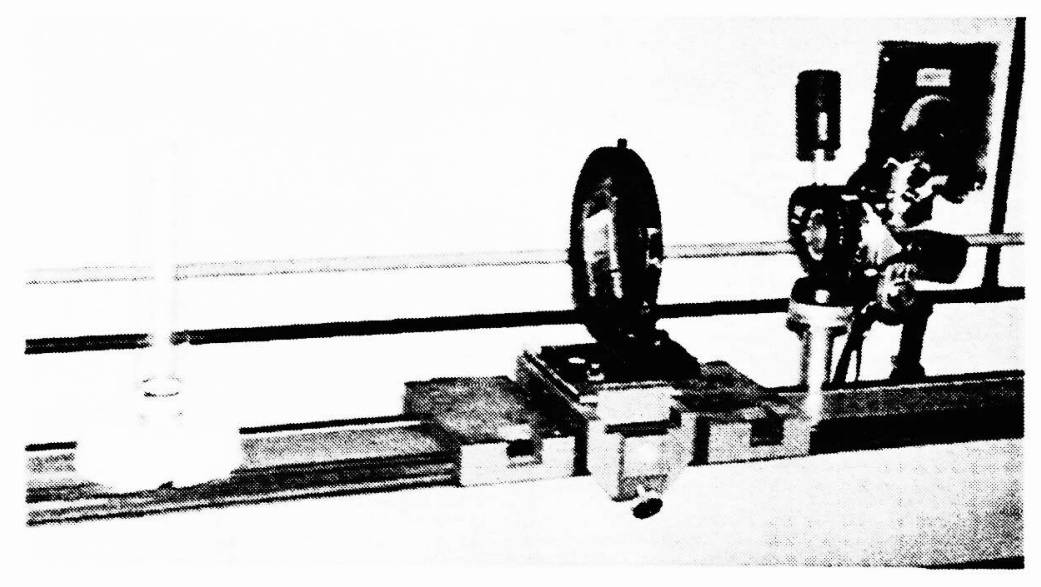

Figure 13. Young's experimental set up with linear filament lamp

As the filamentary light source is incoherent and quasimonochromatic, it can be described as a linear set of independent point sources. Then the intensity distribution can be calculated as the superposition of individual intensity patterns. Besides. as the filamentary source is larger than the Young's interferometer slits, the interference pattern at the observation plane can be divided into three regions: Central, peripheral and dark ones. The contribution of a point light source located on the filamentary source plane $\left(x_{f} ; y_{f}\right)$ to the observation plane $\left(x_{o} ; y_{0}\right)$, can be expressed as

$$
I\left(x_{o}, y_{o}\right)=\operatorname{senc}^{2} \frac{\pi b}{\lambda}\left(\frac{y_{f}}{L}+\frac{y_{o}}{f}\right) \cdot \frac{1+\cos \frac{2 \pi a}{\lambda}\left(\frac{y_{f}}{L}+\frac{y_{o}}{f}\right)}{2} \cdot H\left(\frac{l}{\lambda R} x_{f}+\frac{l}{\lambda f} x_{o}\right)
$$

The width of the Young's slits is $b=37.5 \mu \mathrm{m}$ and they are a distance $a=237.5 \mu \mathrm{m}$ apart. Slits are $l=22 \mathrm{~mm}$ long. The factor $H$ refers to the diffraction at both ends of slits. As its contributions to the total distributed intensity is not too important. it can be approximated by a rectangle function as in Geometric Optics approximation.

As the length of the filamentary source is almost seven times larger than slits, the light from its central part will greatly contribute to the central region of the interference pattern. Due to the same geometric argument, one the extremes of the source will only contribute to the opposite peripheral region in the interference pattern. Then, the total contribution of the source on the interference pattern will be equal to the integral of $I\left(x_{0}, y_{0}\right)$ along the source. Figure 14 is the result of such calculations. $\Delta$ is a variable related with the position of the part of the source under consideration, its longitude, and the angle of rotation $\varphi$ against the line of slits. The case represented at Figure 14 corresponds to $\varphi=32^{\circ}$. Figure 15 shows a collection of five pictures of interferograms taken at $\varphi=0^{\circ}, 8.5^{\circ}, 12.5^{\circ}, 17^{\circ}$, and $20^{\circ}$. Experimental results are in agreement with the theoretical model.

Two remarks follow this theoretical/experimental study on Young interference with incoherent and quasimonochromatic filamentary source. The first one is related with the simplicity of the theoretical model; it is based on the fact that the total contribution to the interference pattern of a set of incoherent and quasimonochromatic sources is equal to the summation of individual pattern contribution. The second remark is that interference fringes are not parallel to the slits. They are parallel to the light source in case that its length will be larger than slits. 


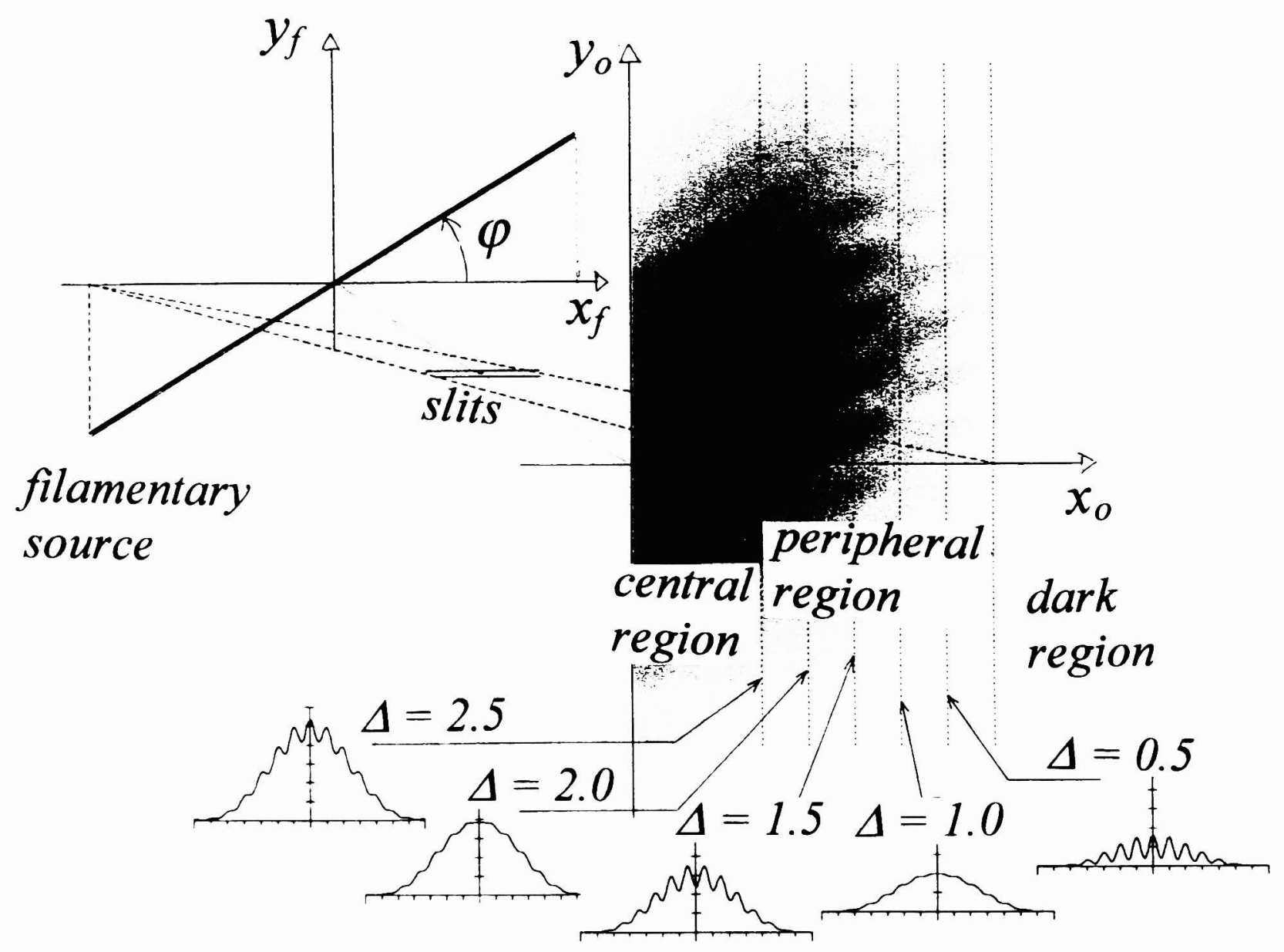

Figure 14. Results of calculations according with the model based on equation (8) for $\varphi=32^{\circ}$

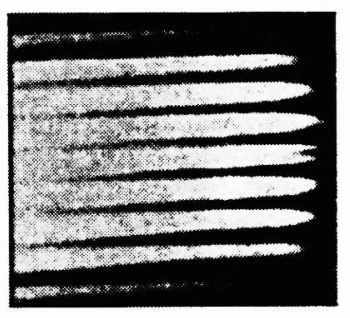

$\varphi=0^{\circ}$

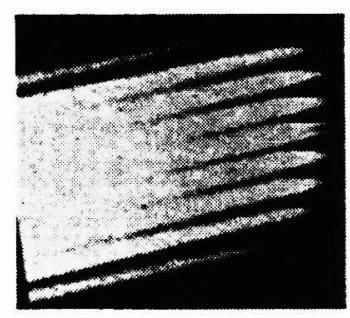

$8.5^{\circ}$

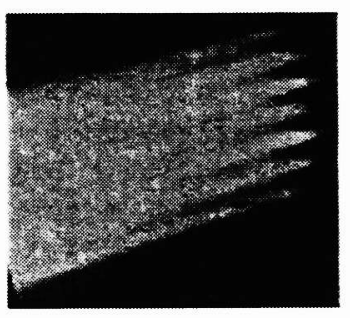

$12.5^{\circ}$

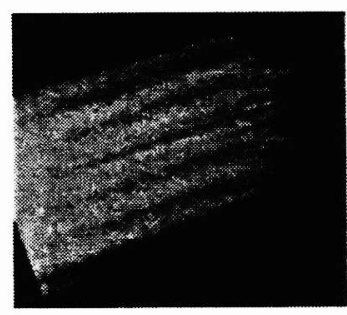

$17^{\circ}$

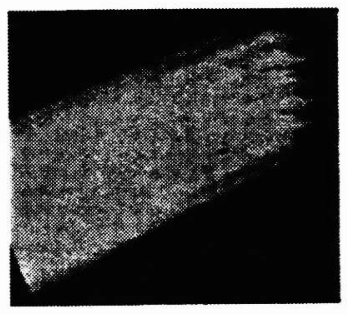

$20^{\circ}$

Figure 15. Collection of five pictures of interferograms taken at $\varphi=0^{\circ}, 8.5^{\circ}, 12.5^{\circ}, 17^{\circ}$, and $20^{\circ}$

\section{CONCLUSIONS}

This paper deals with undergraduate education and training in Optics at the Universidad Nacional de La Plata. Both activities are concentrated in the Optical Laboratory at the Faculty of Astronomy and Geophysics and in the Department of Physics of the Faculty of Exact Sciences. Students in the fourth academic year of the five-year "Licenciado en Astronomia" career and from the sixth to eighth academic semesters of the ten-semester "Licenciado en Física" career, optionally received education and training in Optics. In case of training in Optics at the Optical Laboratory at the Faculty of Astronomy and Gcophysics. technicians and vocational students are also admitted for a one-year course on Optical Instruments. Some theoretical/expenmental works are described 
According with the collected experience, students demonstrated maturity to perform technical and research activities in Optics at 20 to 22 years old under appropriate guidance. They also demonstrated a high level of responsibility to get theoretical and experimental results and to generate a scientific paper for congress presentation or publication.

\section{ACKNOWLEDGEMENTS}

The author is indebted to Prof. Lic. Luis Martorelli, who is director of the Optical Laboratory at the Faculty of Astronomy and Geophysics, for giving him, details of the laboratory activity and pictures, and to K. and G. Videla for helping him in processing images, and to M. Creus for helping him with the edition of the text and figures.

\section{REFERENCES}

1. M. Falabella, L. Giovanetti, M. Mizrahi, L. M. Zerbino and M. Garavaglia, "Measuring light coherence attending to its state of polarization," Paper to be presented at the International Symposium on Laser Metrology for Precision Measurements and Inspection in Industry, Florianópolis, Brazil, October 13-15, 1999.

2. E. C. Cortizo, F. A Videla and M. Garavaglia, "Continuous position detector: Study of retardation plates," Paper to be presented at the International Symposium on Laser Metrology for Precision Measurements and Inspection in Industry, Florianópolis, Brazil, October 13-15, 1999.

3. M. E. Manceñido, G. Pozzi, L. Zunino and M. Garavaglia, "Young interference experiments with a laser filamentary light source," J. Opt. Soc. Am. A. (In press) 1999.

4. M. Born and E. Wolf, Principles of Optics, Pergamon, Oxford ( $5^{\text {th }}$ edition), 1975.

5. M. E. Manceñido, G. Pozzi, L. Zunino, L. M. Zerbino and M. Garavaglia, "Experimentos interferenciales de Young empleando una fuente filamentarua casimonocromática," (In Spanish), Anales de la Asociación Física Argentina, Volumen 10 (In press) 1999. 